\title{
Propuesta docente para la nueva asignatura de Tecnologías Emergentes en el Grado en Ingeniería Informática
}

\author{
Samuel Romero ${ }^{1}$ \\ ${ }^{1}$ Departamento de Arquitectura y Tecnología de Computadores. ETSI Informática y de \\ Telecomunicación. Universidad de Granada \\ sromero@ugr.es
}

\begin{abstract}
Resumen. En este artículo se expone el diseño de la propuesta docente para la nueva asignatura de "Tecnologías Emergentes" que empezará a impartirse en el seno de la especialidad en Ingeniería de Computadores como optativa, en el curso 2013-14. La asignatura, sin precedentes como tal en otras titulaciones similares de nuestro país, tratará de transmitir al alumnado la importancia de permanecer actualizados y familiarizados con las tecnologías en fase emergente. Se enseñará a analizar dichas tecnologías en cuanto a expectativas, viabilidad, y aplicaciones. A modo de ejemplo, se aproximará a los estudiantes a tres tecnologías emergentes: Sistemas vestibles, redes de sensores inalámbricos, e interfaces cerebro-computador. El enfoque global de la asignatura es eminentemente práctico y participativo, buscando dotar al estudiante de las habilidades necesarias para el desempeño de su profesión.
\end{abstract}

Palabras Clave: tecnologías emergentes, sistemas vestibles, redes de sensores inalámbricos, interfaces cerebro-máquina, $\mathrm{BCI}$, Arduino, metodología docente.

\begin{abstract}
In this paper, we depict the design for the teaching proposal for a new course on "Emerging Technologies", to start within the minor on Computer Engineering, in the academic year 2031-14. This subject, without precedents as this one in similar degrees in our country, aims to make the students perceive the relevance of keeping updated and familiarized with respect to technologies in emerging phase. We will train the students in the analysis of such technologies regarding expectancies, viability and applications of them. As examples, we will introduce the students into three emerging technologies: wearable systems, wireless sensors networks, and brain-computer interfaces. The global focusing for the course is mainly practical and interactive, seeking to provide the students with the skills that are required to perform adequately in their professional life.
\end{abstract}

Keywords: emerging technologies, wearable systems, wireless sensors networks, brain-computer interfaces, BCIs, Arduino, teaching methodology. 


\section{Introdución}

En este artículo se presenta una propuesta didáctica para una nueva asignatura del Grado en Ingeniería Informática, denominada "Tecnologías Emergentes". Esta asignatura se impartirá por primera vez en el próximo curso 2013-2014. En los siguientes apartados se realiza una descripción de las características de la asignatura, según se recoge en el plan de estudios. Se realiza una breve panorámica de los contenidos que componen la materia, y su interrelación con otras asignaturas de la carrera y de la especialidad en Ingeniería de Computadores.

Además de contenidos propiamente relacionados con determinadas tecnologías emergentes, y que, por su propia naturaleza, están sujetas a evolución o incluso extinción, se pretende dotar al estudiante de las habilidades necesarias para localizar y detectar tecnologías en fase emergente, analizar su viabilidad y aplicaciones, y ser capaces de familiarizarse rápidamente con éstas.

Se describen, conforme al diseño de la ficha VERIFICA, las competencias y objetivos que se han de cubrir con esta asignatura, y se detallan los contenidos teóricos y prácticos que se han previsto para la misma.

Finalmente, se expone la metodología docente que se pretende emplear, con enfoque novedoso y participativo. Se describe el sistema de evaluación previsto para esta asignatura y se comenta brevemente la bibliografía que se va a emplear.

\section{La nueva asignatura de Tecnologías Emergentes}

\subsection{Contexto}

Con la llegada de los nuevos planes de estudios que se derivan del proceso de Bolonia, en la Escuela Técnica Superior de Ingenierías Informática y de Telecomunicaciones de la Universidad de Granada se ha venido implantando en los últimos años un nuevo título de Grado en Ingeniería Informática.

Este título cuenta con varias intensificaciones o especialidades de rama, entre las que se encuentra la de Ingeniería de Computadores, que mayoritariamente se imparte desde el Departamento de Arquitectura y Tecnología de Computadores de la Universidad de Granada.

En el cuarto y último curso de esta titulación, y dentro de la materia denominada "Complementos de Sistemas de Cómputo para Aplicaciones Específicas", se oferta la materia "Tecnologías Emergentes" [1], con carácter optativo. 
Esta asignatura tiene previsto su comienzo en el primer semestre del curso académico 2013-2014. Si bien está adscrita al Grado en Ingeniería Informática, se puede ofertar también en el Grado en Ingeniería de Tecnologías de Telecomunicación.

Para cursar esta asignatura no se han establecido prerrequisitos obligatorios, si bien sería beneficioso para aquellos estudiantes que la vayan a cursar el haber estudiado antes otras materias como Sistemas con Microprocesador y Sistemas Empotrados, entre otras.

\subsection{Contenidos principales de la asignatura}

Atendiendo a lo recogido en la ficha VERIFICA, los contenidos básicos de la asignatura son los siguientes:

- Hardware para sistemas vestibles.

- Redes de sensores inalámbricos.

- Sistemas para rehabilitación sensorial.

- Sistemas implantables.

- Aplicaciones biomédicas.

- Interfaces cerebro-máquina.

- Conformidad y certificación de la tecnología

Como se puede ver, los contenidos de Tecnologías Emergentes giran, principalmente, en torno a tres tecnologías modernas:

- $\quad$ Sistemas vestibles (Wearable Computing o e-Textiles)

- $\quad$ Redes de Sensores Inalámbricos (Wireless Sensor Networks, IoT, M2M)

- Interfaces Cerebro-Máquina (BCI o BMI, Neuroengineering)

Los sistemas vestibles [2] se refieren a aquellos elementos electrónicos (computador, microcontrolador, sensores, actuadores, etc.) que se pueden incorporar en la vestimenta del usuario, con muy diversos fines. Así, podemos encontrar ejemplos de estos sistemas en ropa "inteligente" que monitorice la salud de un paciente, sistemas de señalización para vestimenta nocturna en carreteras (ciclistas, agentes de tráfico, operarios de mantenimiento), calzado deportivo inteligente que registre nuestra actividad, o complementos para personal militar que faciliten la comunicación con otros y la localización de objetivos.

Todos estos sistemas presentan una serie de requisitos comunes en cuanto al tipo de sistemas que deben incorporar: bajo consumo, fácil integración en elementos textiles, escaso volumen, interfaces integrables y cómodas, etc. 
Por otra parte, las redes de sensores inalámbricos [3] se relacionan con tecnologías en las que elementos de muy bajo consumo, empotrables en distintos dispositivos y con capacidad para hacer interfaz con sensores analógicos o digitales, incluyen la capacidad de crear redes de estos nodos de sensores que se pueden intercomunicar siguiendo diferentes estándares. En esta línea se han generado nuevos términos como "Internet of Things" (IoT) y comunicación M2M ("Machine-to-machine").

Las aplicaciones de estas redes de sensores inalámbricos pueden ir desde la monitorización ambiental de áreas extensas (ciudades, plantas industriales, zonas forestales), domótica, comunicación entre vehículos, entre robots autónomos, etc.

Por último, se contempla el estudio de principios de Neuroingeniería [4] para el desarrollo de sistemas que permitan comunicar el cerebro o el sistema nervioso con un computador (Interfaces cerebro-máquina o cerebro-computador). De esta forma, mediante una serie de electrodos, se puede registrar la actividad mental y usarla para controlar distintos elementos como sillas de rueda, sistemas domóticos, interfaces de comunicación, etc.

\subsection{Un enfoque con vista al futuro}

Si bien los contenidos principales de la asignatura se centran en las tres tecnologías anteriormente mencionadas, el propio carácter de la asignatura se presta a una constante evolución, con la extinción de tecnologías ya superadas y el nacimiento de otras nuevas.

Por tanto, será necesario, por una parte, el actualizar este catálogo de tecnologías emergentes con las que los estudiantes se deben familiarizar. Pero por otra parte, se busca dotar a estos estudiantes de habilidades no tan efímeras como puedan serlo algunas de estas tecnologías.

Nos referimos a la capacidad en sí de detectar nuevas tecnologías en fase emergente, analizar su viabilidad y posibilidades de supervivencia, su aplicabilidad, y en definitiva, el desarrollo del pensamiento crítico necesario en el ámbito de la ciencia y la tecnología, y que resulta imprescindible para discernir el verdadero interés y potencial de las tecnologías según se presentan en diferentes fuentes de información.

De esta forma, buscamos que el estudiante analice tecnologías en el pasado han fracasado a pesar de su potencial (el video Beta, el miniDisc, por ejemplo), el tiempo de vida cada vez más corto de las tecnologías (por ejemplo, discos duros que se van reemplazando por estado sólido), y la fiabilidad de las fuentes de información sobre nuevas tecnologías (por ejemplo, medios de comunicación de masas vs. Revistas especializadas como el MIT Technology Review [5]). 


\section{Diseño formal de la asignatura}

\subsection{Competencias}

Según se recoge en la ficha VERIFICA, en la asignatura de Tecnologías Emergentes se han seleccionado las siguientes competencias para su desarrollo en el alumnado:

\section{- Competencias Específicas de la Asignatura}

IC1. Capacidad de diseñar y construir sistemas digitales, incluyendo computadores, sistemas basados en microprocesador $\mathbf{y}$ sistemas de comunicaciones.

IC5. Capacidad de analizar, evaluar y seleccionar las plataformas hardware y software más adecuadas para el soporte de aplicaciones empotradas y de tiempo real.

IC6. Capacidad para comprender, aplicar y gestionar la garantía y seguridad de los sistemas informáticos.

IC7. Capacidad para analizar, evaluar, seleccionar y configurar plataformas hardware para el desarrollo y ejecución de aplicaciones y servicios informáticos

\section{- Competencias Específicas del Título}

E1. Capacidad para concebir, redactar, organizar, planificar, desarrollar y firmar proyectos en el ámbito de la ingeniería en informática que tengan por objeto, de acuerdo con los conocimientos adquiridos, la concepción, el desarrollo o la explotación de sistemas, servicios y aplicaciones informáticas. E4. Capacidad para definir, evaluar y seleccionar plataformas hardware y software para el desarrollo y la ejecución de sistemas, servicios y aplicaciones informáticas.

E7. Capacidad para conocer, comprender y aplicar la legislación necesaria durante el desarrollo de la profesión de Ingeniero Técnico en Informática y manejar especificaciones, reglamentos y normas de obligado cumplimiento E8. Conocimiento de las materias básicas y tecnologías, que capaciten para el aprendizaje y desarrollo de nuevos métodos y tecnologías, así como las que les doten de una gran versatilidad para adaptarse a nuevas situaciones.

\subsection{Objetivos}

Se pretende que los estudiantes que hayan cursado con éxito la asignatura de Tecnologías Emergentes, sean capaces de: 
- Analizar las nuevas tecnologías en Ingeniería de Computadores. Detectar tendencias emergentes en fase de investigación. Conocer las líneas de investigación en auge en Ingeniería de Computadores. Familiarizarse con estas novedades tecnológicas.

- Caracterizar un sistema vestible, identificar sus requisitos en función de la aplicación concreta y diseñar un sistema completo. Identificar ejemplos de sistemas vestibles en fase de investigación y desarrollo.

- Conocer, concebir y desplegar redes de sensores inalámbricos. Comprender las tecnologías de comunicación inalámbrica y los protocolos usados. Detectar aplicaciones en las que estas redes puedan ser de utilidad.

- Conocer principios básicos de ingeniería biomédica. Comprender el papel de la Ingeniería de Computadores en biomedicina.

- Familiarizarse con las técnicas de tratamiento de información biológica, en especial con señales del sistema nervioso.

- Clasificar las interfaces cerebro-máquina, conocer los sistemas de referencia para la obtención de señales cerebrales, identificar los distintos tipos de señales y su posible modulación mediante la voluntad, y definir el sistema de clasificación de señales y/o patrones cerebrales.

- Conocer la normativa y certificaciones que han de cumplir dispositivos como los que se estudian en la asignatura (sensores inalámbricos, implantes activos, dispositivos biomédicos).

\subsection{Contenidos}

El programa de contenidos concretos previstos para la asignatura, contempla los siguientes temas:

Tema 1. Sistemas Vestibles.

1.1. Computación Ubicua y Vestible

1.2. Aplicaciones de los sistemas vestibles.

1.3. Hardware para sistemas vestibles.

Tema 2. Redes de sensores.

2.1. Sensores y redes. Arquitectura de red.

2.2. Esquemas de programación.

2.3. Estándares y sistemas actuales

2.4. Aplicaciones de las redes de sensores.

Tema 3. Sistemas de Rehabilitación Sensorial.

3.1. Ingeniería de la Rehabilitación

3.2. Robótica Asistencial

3.3. Sustitución y apoyo sensorial

Tema 4. Sistemas Implantables. Aplicaciones Biomédicas

4.1. Principios de Ingeniería Biomédica. 
4.2. Implantes y sistemas implantables.

4.3. Aplicaciones biomédicas implantables.

Tema 5. Interfaces Cerebro-Máquina.

5.1. Concepto y tipos.

5.2. ICM no invasivos.

5.3. ICM invasivos.

5.4 Ejemplos.

Tema 6. Conformidad y Certificación de la Tecnología

6.1 Regulación y certificación de redes de sensores

6.2 Organismos certificadores de sistemas implantables

6.3 Certificación de implantes y sistemas biomédicos.

\section{Metodología docente}

\subsection{Clases teóricas}

Las clases de teoría de la asignatura se organizarán en dos fases. En la primera fase, se combinarán las lecciones magistrales habituales para sentar los conceptos básicos con la puesta en común de trabajo autónomo del alumnado. Este trabajo autónomo estará previamente preparado a partir de una serie de guías (se incluye un ejemplo como anexo) que orientarán al estudiante en la aproximación al tema que se va a impartir, de manera que pueda averiguar por su cuenta conceptos, ejemplos y técnicas que luego podrá compartir y discutir con el resto de la clase. Esta técnica hará las clases teóricas más participativas, evitando el clásico monólogo del profesor, a la vez que permitirá potenciar la capacidad de aprendizaje autónomo del estudiante, tan necesaria en una profesión que requiere constante actualización y más en el curso previo a su incorporación al mercado laboral.

Como anexo a este artículo, se plantea un ejemplo de guía de trabajo autónomo, escrito en un lenguaje accesible, con dificultad progresiva y que pretende motivar al estudiante en su aproximación activa al próximo tema que se ha de ver en clase.

Además, en esta primera fase, se intercalarán una serie de seminarios:

- Seminario 1: análisis crítico de la información

- Seminario 2: fuentes de información sobre tecnologías emergentes

- Seminario 3: Arduino, fundamentos básicos

- Seminario 4: Android (en función de conocimientos iniciales)

- Seminario 5: cómo hacer una presentación (ídem) 
Una vez concluida esta primera fase, se dedica la última parte de la asignatura a una segunda fase en la que los estudiantes realizan presentaciones ante la clase acerca de una tecnología en fase emergente. Esta presentación versará sobre un tema previamente seleccionado y supervisado en una tutoría específica. Deberá incluir una aproximación a esta tecnología, así como un análisis de la misma (fundamentos, viabilidad, expectativas, aplicaciones, fuentes de información).

\subsection{Clases prácticas}

Las sesiones de prácticas de la asignatura se organizarán también en dos fases. En la primera fase, se realizarán una serie de prácticas-tutoriales basados en guiones, al objeto de que los estudiantes se familiaricen con el uso básico de estas tecnologías. Se proponen las siguientes prácticas guiadas:

- $\quad$ Práctica 1: Sistemas vestibles: hardware para dispositivos vestibles, basados en LillyPad Arduino [6]. Demostración de montaje y programación de un chaleco con señalización nocturna basado en microcontrolador.

- Práctica 2: Redes de sensores inalámbricos: montaje de un sistema basado en comunicación entre motas inalámbricas basado en XBee [7] y Arduino

- Práctica 3: Uso de plataformas BCI bajo coste: Neurosky Mindset [8] y Emotiv EPOC [9] (registro de señales de actividad cerebral, y programación para control de un dispositivo).

En la segunda fase, los estudiantes, por grupos cuya composición dependerá del número total de estudiantes, trabajarán en un pequeño proyecto libre basado en la aplicación de alguna de las tecnologías vistas en la primera fase o incluso una combinación de ellas. La selección de este proyecto será monitorizada en tutoría previa, y se hará un seguimiento de su desarrollo en las sesiones de prácticas. Al finalizar, los estudiantes presentarán a la clase sus resultados y conclusiones.

\subsection{Método de evaluación}

Para la evaluación de los estudiantes que sigan esta asignatura, se ha planteado el uso de los siguientes mecanismos y su ponderación en la calificación final:

- La nota final se compondrá de: Teoría $(50 \%)+$ Prácticas $(50 \%)$

- Teoría

- Participación en clase de teoría (resultado del trabajo autónomo) $10 \%$

- $\quad$ Tests elaborados colaborativamente (al final de cada tema) $20 \%$

- Exposición final sobre una tecnología (usando una rúbrica) 20\% 
- Prácticas

- Asistencia a sesiones prácticas $20 \%$

- Exposición del proyecto desarrollado (usando una rúbrica) $30 \%$

De esta forma, se estimula la participación del estudiante en el desarrollo de la asignatura, y se le hace co-partícipe de la evaluación, dado que intervendrá proponiendo preguntas de test para su inclusión en una base de cuestiones de las que se alimentarán automáticamente los tests de cada tema, que se harán por ordenador. Este esquema ha sido ensayado anteriormente con muy buenos resultados [10].

Es muy conveniente el uso de "rúbricas" [11] o plantillas de criterios de evaluación, de los que dispondrán con antelación los estudiantes, de manera que sepan qué deben hacer para obtener una determinada calificación, y qué aspectos se deben cuidar para sus trabajos (por ejemplo, en la exposición del proyecto, atenerse al tiempo preestablecido para sacar la máxima calificación, o no leer las transparencias).

Además de la evaluación habitual a la que son sometidos los estudiantes, se incluirán mecanismos para evaluar la acción del docente y el diseño de la asignatura, de cara a proporcionar una retroalimentación que redunde en una mejor docencia. En condiciones ideales, se realizará una encuesta en un punto intermedio del semestre (de forma que las posibles mejoras puedan aplicarse en ese mismo curso) y otra al final (para perfilar el diseño de cara al curso siguiente).

\subsection{Bibliografía}

La bibliografía que se empleará en esta asignatura se ha seleccionado teniendo en cuenta que se trate de obras prácticas y asequibles desde el punto de vista de su lectura, y que tengan una total disponibilidad para los estudiantes.

Por tanto, hemos optado por títulos que están disponibles en formato electrónico en el catálogo de la Biblioteca de la Universidad de Granada. Esto conlleva dos ventajas: por una parte, el coste cero para el estudiante, y por otra la disponibilidad que nos evita las restricciones típicas de un número limitado de copias en papel.

Dado que están en su último curso de grado, es conveniente que se maneje la bibliografía en inglés, tal y como se ha seleccionado. Todos los libros se basan en ejemplos y proyectos con la placa Arduino, por lo que son prácticos y nos permiten ilustrar ejemplos teóricos e inspirar diseños para el laboratorio de prácticas.

Adicionalmente, se ofrecerá una selección de títulos para profundizar de manera opcional (protocolos, fundamentos, etc.). $[2,3,4]$ 


\section{Referencias}

1. Universidad de Granada. Tecnologías Emergentes, guía docente. Disponible on-line en http://grados.ugr.es/informatica/pages/infoacademica/guias_docentes/optespic/tecnologiase mergentes?pagina $p d f=1(2013)$

2. Barfield, W., Caudell, T, (Eds.). Fundamentals of Wearable Computers and Augmented Reality. CRC Press, New Jersey (2001)

3. Sohraby, K. Miloni, D, Znati, T. Wireless Sensor Networks. Technology, protocols and applications. Wiley, New Jersey (2007)

4. Akay, M. Handbook of Neural Engineering. Wiley, New Jersey (2007)

5. Massachussets Institute of Technology. MIT Technology Review. Disponible on-line en http://www.technologyreview.com/ (2013)

6. Arduino. Placa "LillyPad". Disponible on-line en http://arduino.cc/es/Main/ArduinoBoardLilyPad (2013)

7. Digi, Inc. Módulos XBee. Disponible on-line en http://www.digi.com/xbee/ (2013)

8. Neurosky Inc. MindSet. Disponible on-line en http://www.neurosky.com/Products/MindSet.aspx (2013)

9. Emotiv. EPOC headset. Disponible on-line en http://www.emotiv.com/apps/epoc/299/ (2013)

10. Romero, S. y A. Guillén. Evaluación y Autoevaluación Continuas Automatizadas Mediante Herramientas de Software Libre. Actas del I Congreso de Docencia Universitaria. Universidad de Vigo. pp. 259-264 (2009)

11. H. Andrade, Y. Du, Student perspectives on rubric-referenced assessment, in Practical Assesment, Research \& Evaluation, vol. 10, n³ 3 pp. 1-11. (2005)

12. Olsson, T. Arduino wearables. Apress. Berkeley, Calif., (2012)

13. Faludi, R. Building wireless sensor networks. O'Reilly. Beijing, (2011).

14. Igoe, T. Making things talk., O'Reilly. Beijing (2007).

15. Karvinen, T., \& Karvinen, K. Make a mind-controlled Arduino robot. Sebastopol, CA, O'Reilly. http://proquest.safaribooksonline.com/?fpi=9781449312800. (2012)

ANEXO (en páginas siguientes): Ejemplo de Guía de Trabajo Autónomo de Tecnologías Emergentes. 


\section{ANÁLISIS DE TECNOLOGÍAS EMERGENTES}

GUÍA DE TRABAJO AUTÓNOMO DEL TEMA 1

G R A D O E N I N G E N I E R Í A

I N F O R M Á T I C A

$4^{\circ} \mathrm{C}$ U R S O 


\section{Cómo usar esta guía}

Como estudiante de cuarto curso de carrera, tu acceso al mundo profesional se aproxima. A lo largo de la carrera no sólo debes haber adquirido una serie de conocimientos técnicos para el ejercicio de tu profesión, sino que además debes haberte transformado en una persona competente. Esto quiere decir que no debes sólo ser un erudito en Ingeniería Informática, sino que además debes poseer una serie de capacidades ("competencias") que te permitirán desempeñar tu trabajo con éxito (trabajo en equipo, capacidad de comunicación, etc.).

Una de estas competencias esenciales es la capacidad de trabajar de manera autónoma y de seguir formándote por tus propios medios una vez que abandones el ámbito académico. Es lo que se denomina "Life-long Learning", o aprendizaje permanente a lo largo de la vida. Esta competencia es especialmente relevante en nuestra profesión, dada la velocidad con que aparecen nuevos procesadores, lenguajes, dispositivos...

Esta guía te servirá para adquirir de manera autónoma conocimientos que te permitirán afrontar con éxito el estudio del Tema 1 de la asignatura: Análisis de Tecnologías Emergentes. Si sigues los pasos que se detallan en la guía y realizas las actividades, tu éxito en la adquisición de estos conceptos está asegurado. Si además te quedas con ganas de más, haz también las actividades que se marcan como opcionales.

Oigo y olvido. Veo y aprendo. Hago y entiendo.

- Confucio, Filósofo chino (551 AC-478 AC). 


\section{¿Qué vamos a estudiar en este tema?}

A estas alturas de carrera, ya en el último curso, has estudiado multitud de técnicas, métodos, sistemas y herramientas que conforman el cuerpo de esta materia de conocimiento que es la Ingeniería Informática. Si echases un vistazo a planes de estudio anteriores al tuyo, observarás que hay materias que se estudiaban y que tú ya no estudias, y temas nuevos que tus antecesores no tuvieron que estudiar y que a ti te ha tocado aprender. Esta evolución se debe, lógicamente, a que nuestra carrera está en constante renovación (en consonancia con la ciencia y con el mercado), y en el transcurso de pocos años algunas tecnologías llegan a su fin, y otras nuevas surgen.

En este tema vamos a familiarizarnos con las llamadas "Tecnologías Emergentes". En primer lugar, reflexionaremos sobre el concepto de tecnología en sí, y sobre el ciclo de vida de una tecnología. Analizaremos los factores que influyen en el desarrollo de una tecnología, así como en su éxito o fracaso. Veremos algunos ejemplos de tecnologías informáticas del pasado y apuntaremos a algunas de las nuevas tecnologías que emergen recientemente.

En segundo lugar, y en correspondencia con un primer seminario de la asignatura, aprenderemos a distinguir la calidad de las fuentes de donde obtenemos información acerca de estas tecnologías en fase emergente. Para ello, hablaremos de fiabilidad de las fuentes, analizaremos el tratamiento que se hace de la tecnología en los medios de comunicación, y aprenderemos técnicas para discriminar las informaciones verídicas de las carentes de fundamento. 


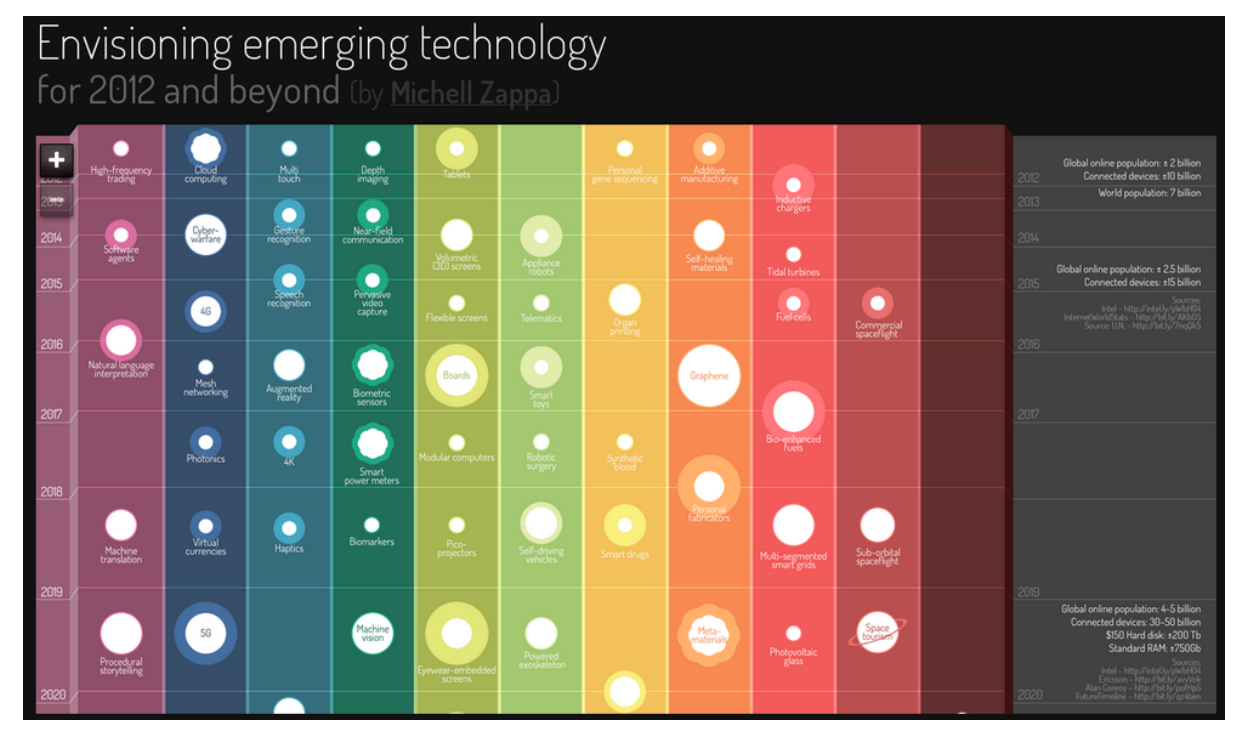

En este segundo bloque se pretende que desarrolles una competencia de especial valor, no sólo para esta materia o para tu desempeño en la vida profesional, sino para ti mismo en todos los aspectos: el pensamiento crítico.

En el seminario que acompaña al tema 1, aprenderemos a analizar argumentos y sentencias para detectar aquellas que no son válidas (falacias). Esto nos permitirá filtrar las fuentes de donde recoger información sobre tecnologías emergentes. Además, esta competencia te permitirá expresarte mejor, ya que aprenderás también a aplicar estas técnicas a tus propios razonamientos.

El tema continúa distinguiendo fuentes formales (científicas) de informales (medios de comunicación de masas, e Internet), y aprenderemos a realizar búsquedas en ambos ámbitos.

Finalizaremos este tema realizando reflexiones sobre el impacto ético que estas tecnologías potenciales pueden tener.

Los conocimientos que adquieras en este primer tema te serán de gran utilidad en tu trabajo final de la asignatura, en el que realizarás un análisis y presentación ante la clase de una tecnología emergente. 


\section{Actividades}

1. Busca en el diccionario de la Real Academia el término "tecnología". ¿en qué se diferencia de "técnica"? ¿y de "ciencia"? Trata de elaborar una definición con tus propias palabras. Pon algunos ejemplos.

2. Mirando atrás en el tiempo, recordarás que han surgido multitud de tecnologías que se prometían superiores a lo vigente, y que sufrieron una "muerte prematura". Un par de ejemplos podrían ser el sistema de video Beta o el MiniDisc. ¿por qué crees que no triunfaron? Prepara tus argumentos para discutirlos en clase.

3. Localiza otros ejemplos de tecnologías que en su momento emergieron pero fracasaron frente a las tecnologías establecidas.

4. (opcional): a modo de "divertimento", trata de encontrar videos o anuncios antiguos de dispositivos tecnológicos que hoy nos resultan un tanto ridículos (en YouTube, o Google Imágenes, aunque la "teletienda" es una fuente inagotable).

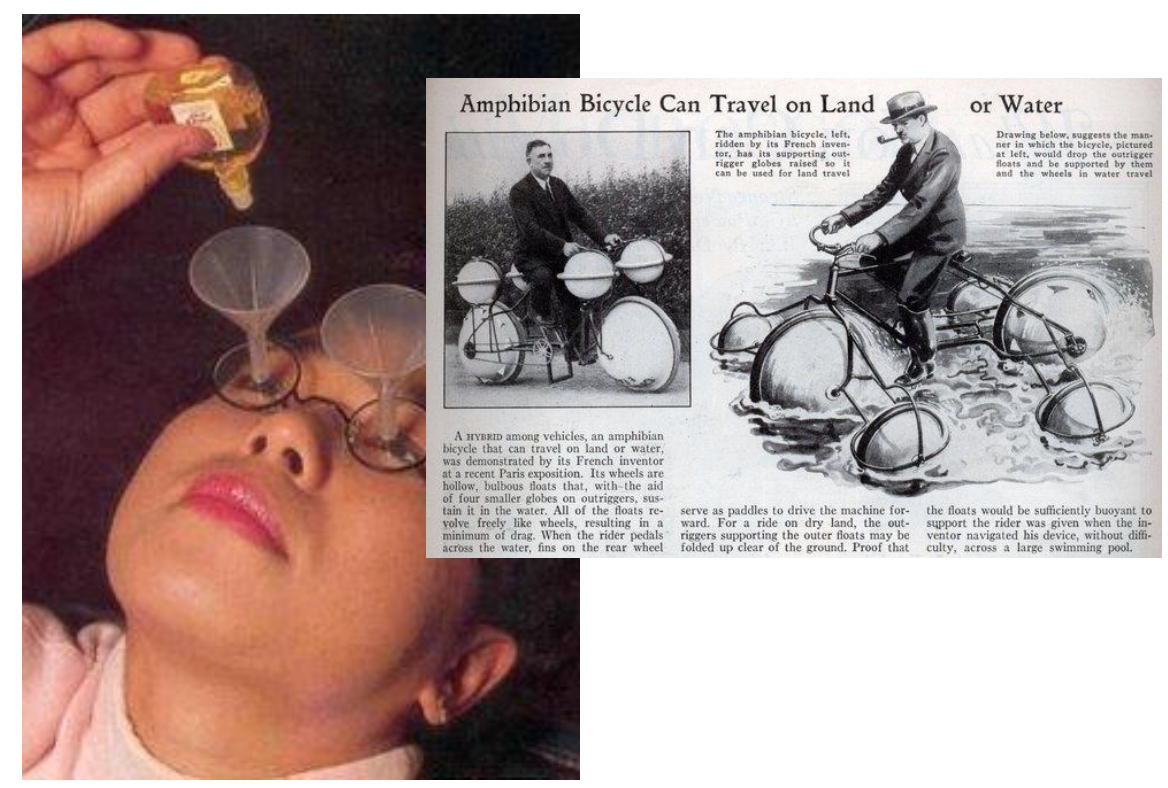


5. Elabora una tabla con tecnologías informáticas que han terminado su ciclo de vida ("legacy"), indicando el nombre de la tecnología en fase de extinción, nombre de la tecnología nueva que la desplaza, y diferencia de prestaciones (por ejemplo, puerto paralelo vs. Puerto USB).

6. ¿la condición esencial para que una tecnología triunfe en el mercado es que ofrezca mejores prestaciones técnicas que sus competidoras? Si es así, justifícalo. Si no, ofrece algún ejemplo de tecnología que, siendo superior a las demás, no ha triunfado (¿por qué?).

7. Una vez realizadas las actividades anteriores, realiza una lectura comprensiva del bloque 1 de transparencias, disponibles en la plataforma virtual. Trata de relacionar los resultados de las actividades que has realizado con los conceptos que se recogen en el tema. Presta especial atención a los factores que influyen en el desarrollo de una tecnología.

8. Escoge una de las tecnologías que hayas aprendido hace poco en la carrera (puede ser un lenguaje de programación, un nuevo tipo de circuito, o una técnica). Trata de averiguar el origen, o la primera vez que se menciona esta tecnología en un documento (pista: intenta localizar fuentes escritas por los inventores).

9. Lee el bloque 2 de transparencias, dedicado a las fuentes de información formales (revistas científicas, bases de datos, patentes) e informales (radio, televisión, prensa, blogs, páginas web y enciclopedias colaborativas).

10. Pon un ejemplo de cada una de las fuentes mencionadas anteriormente.

11. Para una tecnología cualquiera, localiza una fuente informal y otra formal que la describan (pista: localiza primero la noticia, y luego trata de ver qué hay de cierto consultando la fuente científica). ¿qué diferencias observas?

12. (opcional) Echa un vistazo al blog "Malaprensa". Dispone de un apartado en el que se detectan errores de conceptos, datos científicos o mala interpretación estadística, e incluyen la correspondiente explicación: http://www.malaprensa.com

13. Estudia el último bloque de transparencias del tema 1, dedicados al pensamiento crítico, análisis de argumentos y detección de falacias. En el ámbito científico-tecnológico, puede ser útil usar estos "diez mandamientos" para distinguir la ciencia de los camelos (pseudociencias):

I. Basaras tus conocimientos en la evidencia. 
La evidencia en su forma más básica es una observación, así que esta regla implica que las conclusiones deben ser basadas en observaciones. como un detective en la escena del crimen, o en un juicio, las conclusiones están soportadas en evidencias

II. Medirás objetivamente y no supondrás subjetivamente

III. Tus afirmaciones serán respaldadas por evidencia (En otras palabras, decir que algo es un hecho no lo hace un hecho)

IV. Usarás muestras de gran tamaño

V. Tus experimentos serán a ciegas

VI. Tus Experimentos tendrán grupo control

VII. Citarás tus fuentes de información

VIII. Tus fuentes de información serán confiables, verificables y amparadas por la evidencia

IX. La opinión no es un hecho

X. No darás falso testimonio ni mentiras (No harás trampa)

(del blog http://razonesyfalacias.blogspot.com.es/p/ciencia-metodo-ymbe.html).

14. Para el caso de la noticia de la actividad 11 , localiza falacias que no se correspondan con la fuente original. Explica por qué no son afirmaciones válidas.

15. Selecciona una tecnología emergente (por ejemplo, la identificación por radiofrecuencia), y reflexiona sobre los riesgos éticos que presenta. Prepara argumentos para discutirlos en clase.

16. (opcional) Un par de lecturas recomendables para analizar información científica:

- Cómo mentir con estadísticas (Darrel Huff, Ed. Crítica, 2011)

- El mundo y sus demonios (Carl Sagan, Ed. Planeta, 2005)

Vivimos en una sociedad profundamente dependiente de la ciencia y la tecnología y en la que nadie sabe nada de estos temas. Ello constituye una fórmula segura para el desastre.

- Carl Sagan, astrónomo (1934-1996). 